\title{
The Usefulness of Leukosan SkinLink for Simple Facial Laceration Repair in the Emergency Department
}

\author{
Hyunjoo Kim, Junhyung Kim, Jaehoon Choi, Woonhyuk Jung \\ Department of Plastic and Reconstructive Surgery, Keimyung University Dongsan Medical Center, Keimyung University School of Medicine, \\ Daegu, Korea
}

Background Repair of facial laceration in the emergency department can pose a number of difficulties. Children can be uncooperative, but adults can also be if they have sustained head trauma or are intoxicated. Leukosan SkinLink consists of topical adhesive and adhesive tape that can be applied easily to long or tense wounds. In this study, the authors compared conventional suturing with Leukosan SkinLink for facial laceration patients in the emergency department. Methods The prospective study was carried out from March 2013 to September 2013 with linear facial laceration patients visiting the emergency department. Exclusion criteria were open fractures, joint injuries, skin defects, hairy skin, and mucosa. The author used Leukosan SkinLink for skin closure in the experimental group and used conventional suturing in the control group. The scar evaluation using the Patient and Observer Scar Assessment Scale (POSAS) along with satisfaction scores, procedure times, and complications were compared.

Results A total of 77 patients ( 30 in the control group and 47 in the experimental group) participated and underwent follow-up for 6 months postoperatively. The scar assessment using the POSAS and the satisfaction score in both groups were similar. The average procedure time in the experimental group was shorter. In the control group, there were four cases of wound dehiscence, two of infection, and one of skin necrosis, whereas four cases of wound dehiscence and one allergic reaction occurred in the experimental group.

Conclusions With a simple application technique, Leukosan SkinLink is a new effective method for facial laceration repair especially useful for children and uncooperative adults.

Keywords Laceration / Tissue adhesives / Surgical tape / Visual analog scale
Correspondence: Junhyung Kim Department of Plastic and Reconstructive Surgery, Keimyung University Dongsan Medical Center, Keimyung University School of Medicine, 56 Dalseong-ro, Jung-gu, Daegu 700-712, Korea Tel: +82-53-250-7635 Fax: +82-53-255-0632 E-mail: med69@dsmc.or.kr

This article was presented at 72nd Congress of The Korean Society of Plastic and Reconstructive Surgeons on November 7-9, 2014 in Seoul, Korea.

No potential conflict of interest relevant to this article was reported.

Received: 3 Mar 2015• Revised: 5 May $2015 \bullet$ Accepted: 18 May 2015

pISSN: 2234-6163 • elSSN: 2234-6171 • http://dx.doi.org/10.5999/aps.2015.42.4.431 • Arch Plast Surg 2015;42:431-437

\section{INTRODUCTION}

Facial laceration is the most common type of facial trauma for plastic surgeons in emergency departments, and most cases require primary closure. Repair of facial laceration is necessary not only for wound healing without complications but to minimize scarring. However, many problems can arise while suturing facial lacerations, such as uncooperative adults, who may be drunk or have sustained head trauma, uncooperative children, and patients with unstable vital signs. In children especially, apart from scarring, parents are often concerned about the use of sedatives, insufficient anesthesia, and stitch removal. 
With liquefied topical adhesives such as Dermabond (Ethicon, Cincinnati, OH, USA), there is no need for local anesthetics, sedatives, or stitch removal. Hence, the simple procedure is convenient for both physicians and patients and requires less time for outpatient visits. However, these products cannot be applied to long or tense wounds [1]. The purpose of this study was to evaluate Leukosan SkinLink (BSN Medical GmbH, Hamburg, Germany), which consists of liquefied topical adhesive and adhesive tape, and to assess the short- and long-term outcomes of facial laceration repair using this product with regard to cosmesis and postoperative complications for comparison with conventional suturing.

\section{METHODS}

\section{Subjects}

Following Institutional Review Board approval (DSMC 201312-022-004), patients who visited the emergency department of our institution with facial lacerations between March 2013 and September 2013 were evaluated for study inclusion. Written informed consent was obtained from all patients included in this study. Exclusion criteria included the following: known allergy to cyanoacrylates, formaldehyde, or adhesive strips; known dermatosis; impaired wound healing problems such as keloid or rheumatologic disease; steroid or antimetabolic medication; open fractures, joints, or skin defects that require high tension for repair; or any hairy skin or mucosa. Patients who were unconscious or had unstable vital signs owing to multiple traumas were also excluded. Eighty-nine out of 782 patients were included in this study, and patients were allowed to choose the skin closure material; Thirty-eight patients those treated with Leukosan SkinLink were the experimental group and fifty-one patients those who underwent conventional suturing were the control group.

\section{Operation technique}

In the control group, after sterilizing the wound and surrounding skin with $0.05 \%$ chlorhexidine solution, 1:100,000 epinephrine mixed with $1 \%$ lidocaine was infiltrated near the wound. After waiting a few minutes for the hemostatic effect on the wound, simple interrupted skin suture using a non-absorbable suture (6-0 Dafilon, B. Braun, Bethlehem, PA, USA) was performed. If there was muscle injury or the tension needed for repair was high, a simple buried suture on the muscle or subcutaneous tissue using an absorbable suture (5-0 Monosyn, B. Braun) was performed before skin suturing. If the wound margin was dirty and uneven, conservative debridement was performed using a No. 15 blade knife.
In the experimental group, after sterilizing and drying with gauze, adhesive tape was applied perpendicularly to the wound approximating edge with the fingers assisted by the forceps to produce eversion. Liquefied topical adhesive was then applied on the tape rather than directly to the wound. Similar to the control group, debridement or buried suturing was performed if necessary prior to application of Leukosan SkinLink (Fig. 1).

After skin closure in both groups, a semi-occlusive dressing with foam (Mepilex Lite, Molnlycke Health Care, Gothenburg,

\section{Fig. 1. Applying Leukosan SkinLink}

(A) Forehead laceration involving frontalis injury. (B) The frontalis muscle was repaired with 5-0 Monosyn. (C) Adhesive tape was applied perpendicularly to the wound. Liquefied topical adhesive was applied on the tape.
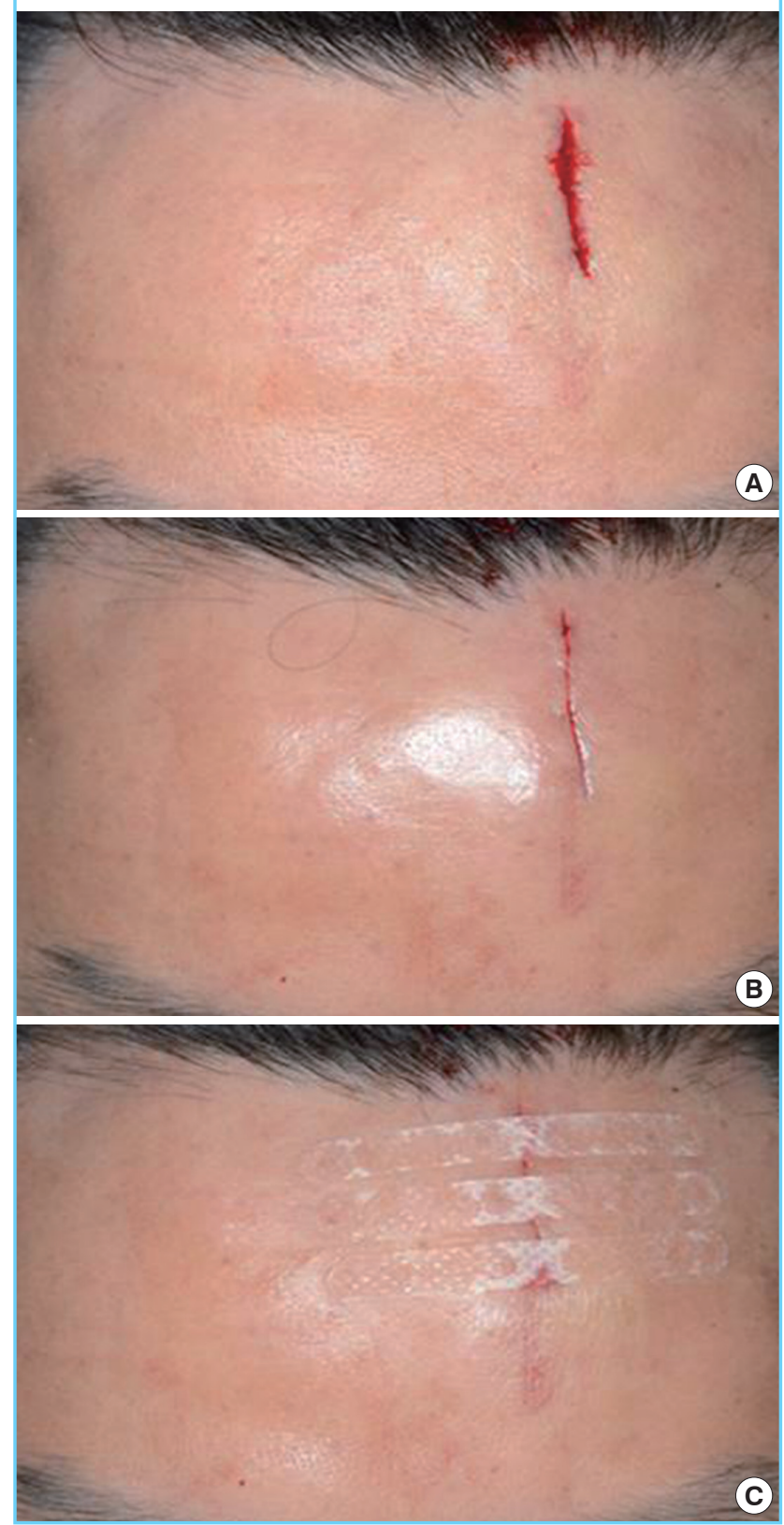
Sweden) was applied. The stitches were removed on postoperative day five in the control group. In the experimental group, the Leukosan SkinLink was allowed to loosen without assistance and was completely removed on postoperative day ten. SteriStrip (3M, Maplewood, MN, USA) was applied in both groups for three months for scar management.

All the series of the procedures in both groups were performed by one of the co-authors (H.J.K.) to exclude the possibility of inter-operator variability.

\section{Evaluation}

The scar was assessed using the POSAS on the day of suture or Leukosan SkinLink removal, then one and six months postoperatively (Fig. 2). The Observer Scale included vascularity, pigmentation, thickness, relief, and pliability, while the Patient Scale included pain, itching, color, stiffness, thickness, and irregularity. Each item could be rated between 1 and 10, with a score of 10 reflecting the worst imaginable scar or sensation. The observer scale was assessed by two plastic surgeons facing the patient directly, and the patient scale was assessed by the patients or their parents. The total score for the observer scale was obtained by adding the scores of each of five items (range, 5 to 50) and the total score for the patient scale was obtained by adding the scores of each of six items (range, 6 to 60). The lowest scores, 5 in the observer scale and 6 in the patient scale, were for normal skin [2]. On the day of suture or Leukosan SkinLink removal, patients were asked to assess their overall satisfaction level including comfort as well as pain on suture or Leukosan SkinLink removal using a scale of 1 to 4 ( 1 at one end representing dissatisfaction and 4 at the opposite end representing very satisfactory). The length of the wound and the time required for the procedure, beginning with sterilization and ending with dressing, were compared. Complications were documented by the physician.

\section{Statistical analysis}

Using IBM SPSS Statistics ver. 22.0 (IBM Corp. Armonk, NY, USA) for Windows to analyze identity between the two groups, the chi-squared test was conducted for categorical variables such as gender and location of the wound, and the two-sample t-test and Wilcoxon rank sum test were conducted for the other continuous variables. A P-value of $<0.05$ was considered to indicate statistical significance.

\section{RESULTS}

\section{Subjects}

From a total of 782 patients that visited the emergency department for facial laceration, 89 (control group 38, experimental

\section{Fig. 2. Patient and Observer Scar Assessment Scale (POSAS)}

The observer scale was assessed by the plastic surgeon, and the patient scale was assessed by the patients or their parents. A lower score meant less scarring.

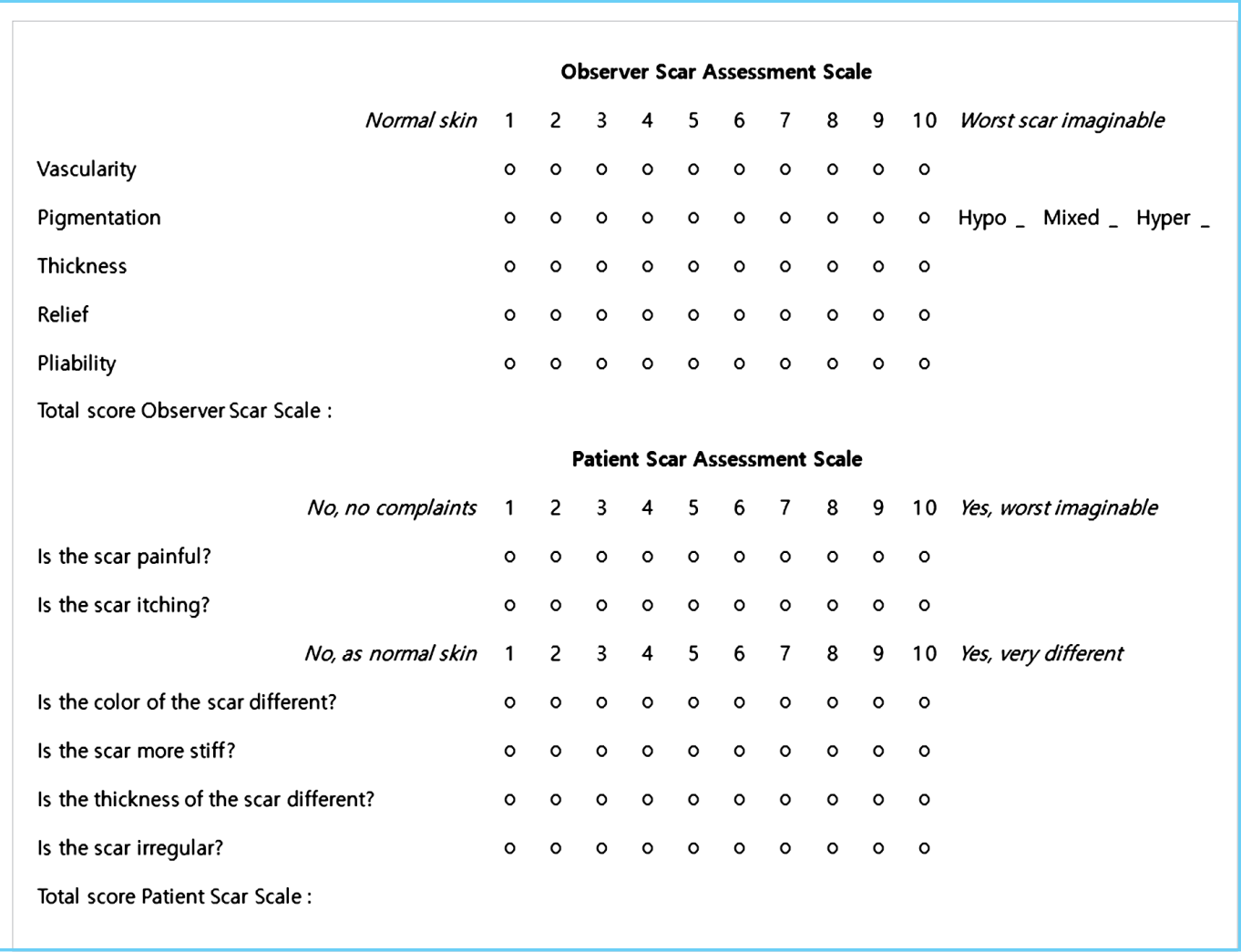


group 51) agreed to participate in this study, among whom 12 patients were lost to follow-up, leaving 77 (control group 30, experimental group 47) who were followed for at least 6 months. Patient demographics in the two groups were not significantly different (Table 1). Among the wound regions, the forehead (control group 17, experimental group 25) was the most common, followed by the chin (control group 9, experimental group 14), cheek (control group 2, experimental group 5), and other sites (control group 2, experimental group 3).

\section{Outcomes}

Scar assessment for the control group by both observers and patients resulted in lower values than for the experimental group. However, other than at one month after surgery $(\mathrm{P}=0.031)$, there was no significant difference between the two groups $(\mathrm{P}>$ 0.05) (Table 2).

The satisfaction score for the procedure was higher in the experimental group, but the difference was not significant $(\mathrm{P}=0.073)$.

\begin{tabular}{|lccc|}
\hline Table 1. Patient demographics \\
\hline Characteristic & $\begin{array}{c}\text { Control } \\
\text { group }\end{array}$ & $\begin{array}{c}\text { Experimental } \\
\text { group }\end{array}$ & P-value \\
\hline No. of patients & 30 & 47 & 0.545 \\
$\quad$ Male & $20(66.7)$ & $32(68.1)$ & \\
$\quad$ Female & $10(33.3)$ & $15(31.9)$ & \\
Age (yr) & $13.67(18.85)$ & $13.57(18.85)$ & \\
Length of wound (cm) $)^{\text {a) }}$ & $1.81(0.84)$ & $1.78(0.77)$ & \\
No. of patients with sedation & $15(50)$ & $5(10.6)$ & $<0.001$ \\
Depth of wound & & & 0.270 \\
Muscle injury (-) & $22(73.3)$ & $30(63.8)$ & \\
Muscle injury (+) & $8(26.7)$ & $17(36.2)$ & \\
Location of wound & $17(56.7)$ & $25(53.2)$ & 0.948 \\
Forehead & $9(30.3)$ & $14(29.8)$ & \\
Chin & $2(6.7)$ & $5(10.6)$ & \\
Cheek & $2(6.7)$ & $3(6.4)$ & \\
Other & & \\
\hline Values except age and length are presented as number (\%). & \\
alPresented as mean (standard deviation). & & \\
\hline
\end{tabular}

\begin{tabular}{|c|c|c|c|}
\hline Characteristic & $\begin{array}{l}\text { Control } \\
\text { group }\end{array}$ & $\begin{array}{l}\text { Experimental } \\
\text { group }\end{array}$ & P-value \\
\hline \multicolumn{4}{|l|}{ Patient score } \\
\hline Immediately postoperative & $19.5(9.7)$ & $20.87(9.6)$ & 0.545 \\
\hline Postoperative one month & $18.66(8.34)$ & $21.1(9.39)$ & 0.249 \\
\hline Postoperative six months & $20.43(8.79)$ & $20.78(8.5)$ & 0.860 \\
\hline \multicolumn{4}{|l|}{ Observer score } \\
\hline Immediately postoperative & $19.16(7.34)$ & $21.85(7.7)$ & 0.133 \\
\hline Postoperative one month & $18.8(7.92)$ & $22.8(7.79)$ & 0.031 \\
\hline Postoperative six months & $17.23(6.2)$ & $18.74(5.81)$ & 0.282 \\
\hline
\end{tabular}

The procedure time per centimeter for the experimental group was 16.95 minutes shorter with sedation and 12.01 minutes shorter without sedation $(\mathrm{P}<0.05)$ (Table 3$)$.

In the control group, four cases of wound dehiscence, two cases of skin necrosis, and two cases of stitch abscesses were reported. In the experimental group, four cases of wound dehiscence and one case of allergic dermatitis were reported. None of the wound dehiscence cases involved full-thickness dehiscence, and they all healed well after re-approximation with the Steri-Strip. The two cases of superficial skin necrosis were less than $5 \mathrm{~mm}$ across and healed completely within two weeks after dressing with foam (Mepilex Lite). The two cases of stitch abscess were treated with incision and drainage. The patient with allergic dermatitis at the periphery of normal skin responded to a topical steroid.

\section{Case 1}

A 62-year-old woman visited the emergency department for a $1.5-\mathrm{cm}$ eyebrow laceration caused by violence. Buried suturing was not performed because the depth of the wound was just beneath the dermis, and the tension to the wound edge was low. Leukosan SkinLink was applied to the wound with manual approximation of the wound edge. There were no complications during a 6-month follow-up, and the patient was satisfied with the cosmetic outcomes (Fig. 3).

\section{Case 2}

A 14-month-old girl visited the emergency department with her parents for a $1-\mathrm{cm}$ forehead laceration sustained during a fall. Buried suturing was not performed, and the wound was easily closed using Leukosan SkinLink without tension. There was no need for sedation. There were no complications during a 6-month follow-up, and the parents indicated that they were satisfied with the simple procedure without sedation and that the scar was acceptable (Fig. 4).

\begin{tabular}{|c|c|c|c|}
\hline Characteristic & $\begin{array}{l}\text { Control } \\
\text { group }\end{array}$ & $\begin{array}{l}\text { Experimental } \\
\text { group }\end{array}$ & P-value \\
\hline Total procedure time (min) & $32.16(6.35)$ & $12.1(7.38)$ & $<0.001$ \\
\hline $\begin{array}{l}\text { Procedure time per } \\
\text { centimeter }(\mathrm{min} / \mathrm{cm})\end{array}$ & $22.16(12.26)$ & $7.12(3.06)$ & $<0.001$ \\
\hline With sedation & $25.35(11.5)$ & $8.4(3.2)$ & $<0.001$ \\
\hline Without sedation & $18.97(12.55)$ & $6.96(3.05)$ & $<0.001$ \\
\hline Satisfaction score & $2.9(0.71)$ & $3.19(0.77)$ & 0.073 \\
\hline Complications (\%) & $8(26.7)$ & $5(10.6)$ & 0.094 \\
\hline
\end{tabular}




\section{Fig. 3. Patient case I}

(A) A 62-year-old woman visited the emergency department after being injured in a violent attack. She sustained a laceration $1.5 \mathrm{~cm}$ in length involving the skin and the subcutaneous layer at the forehead-eyebrow junction. (B) Leukosan SkinLink was applied to the wound despite the presence of the eyebrows in the area. (C) Postoperative 6-month photograph. The patient was satisfied with the scar.
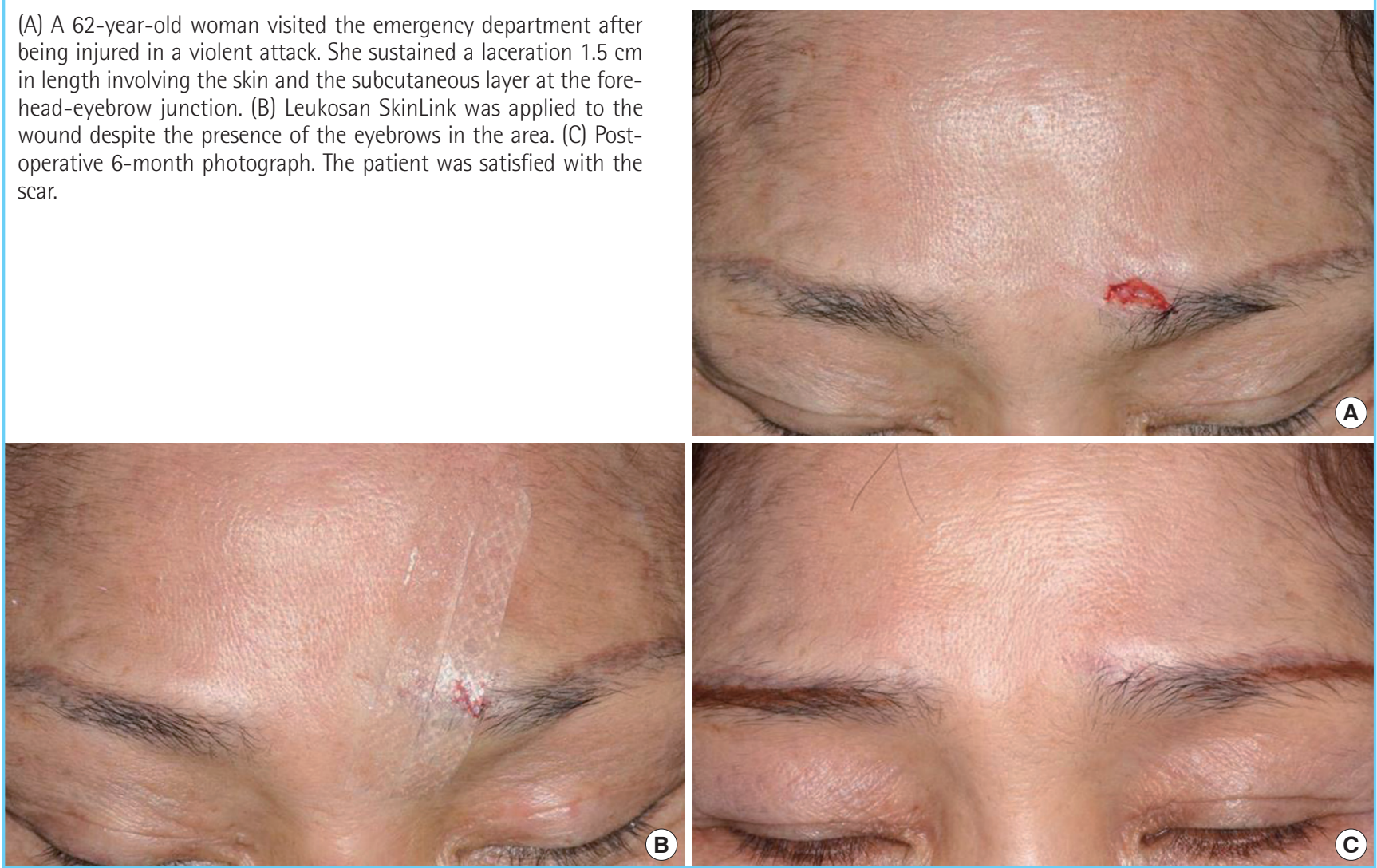

\section{Fig. 4. Patient case II}

(A) A 14-month-old girl was brought to the emergency department with her parents after falling down. She sustained a laceration $1 \mathrm{~cm}$ in length involving the skin and the subcutaneous layer on the forehead. (B) The wound was easily repaired with Leukosan SkinLink without any sedation. (C) Postoperative 6-month photograph. Her parents were satisfied with the scar and the simplicity of the procedure without sedation.

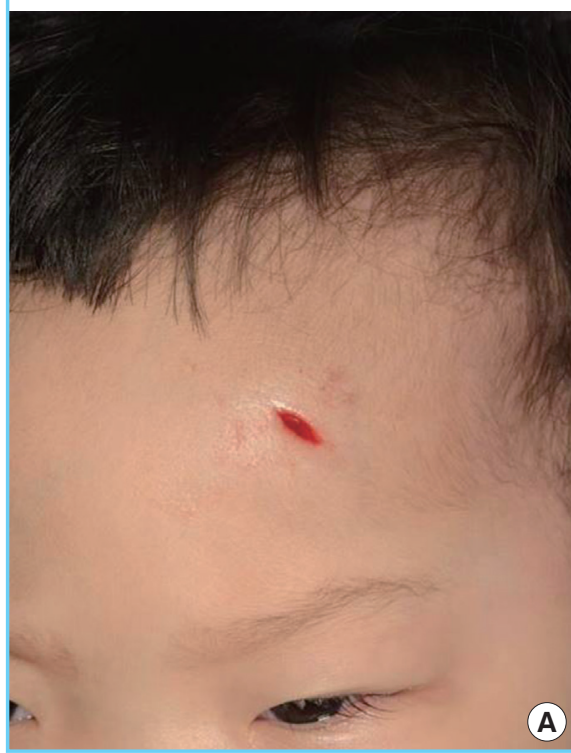

\section{DISCUSSION}

The conventional suture technique is most common because it is
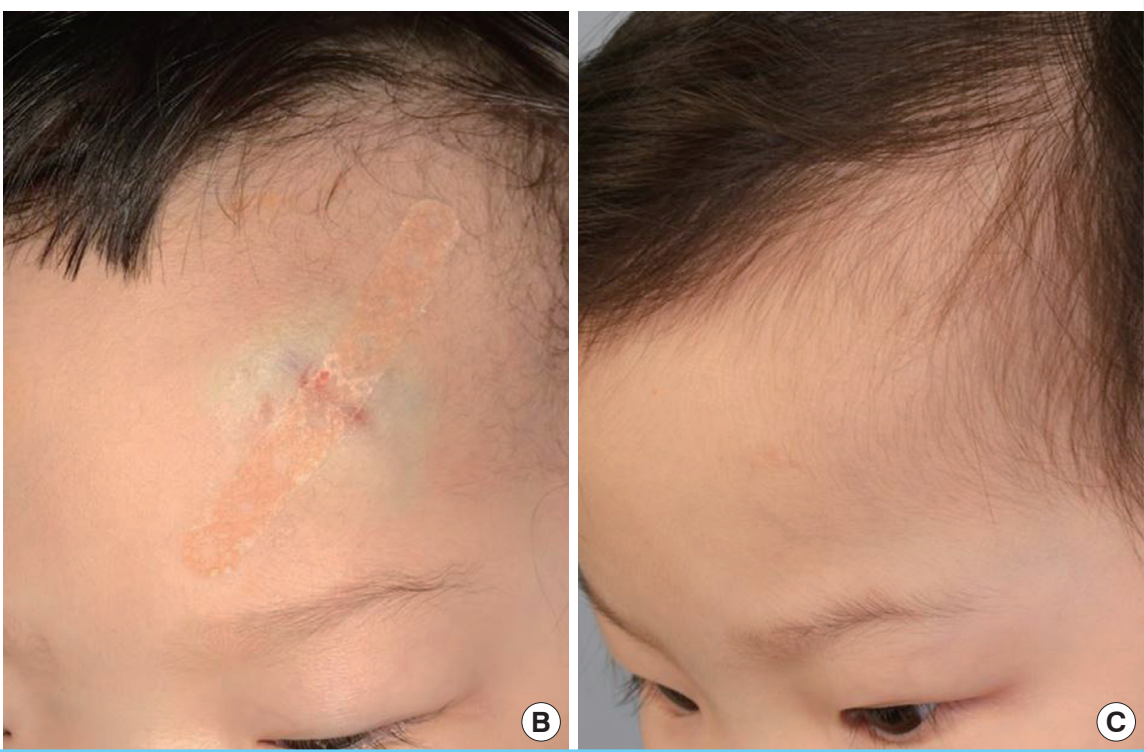

cheap, easy to use for eversion of the wound edge, and has fewer limitations according to the region. However, it requires the use of local anesthetics and potentially, additional sedatives with un- 
cooperative children. In addition, the surgeon may have concerns about the foreign body reaction to the suture material, stitch marks may remain, and patients have to endure the discomfort of stitch removal. Especially in children, due to their behavioral and developmental characteristics, treatment must be not only brief and painless, but also result in acceptable cosmetic outcomes $[3,4]$. To achieve this goal, many materials have been developed and reported. Surgical adhesive tape (Steri-Strip Skin Closure) is simple and easy to use for wound closure but can be removed by sweat and moisture. Liquefied topical adhesive (Dermabond, Histoacryl) is also easy to use and has more tensile strength than surgical tape. However, eversion of the wound edge is difficult to achieve and epithelialization is delayed if the liquid infiltrates to the dermis [1].

Leukosan SkinLink consists of liquefied topical adhesive and adhesive tape. Approximating the wound edge with the fingers, the adhesive tape is applied perpendicularly for tension-free closure. Then, to reinforce the tension-free effect, liquefied topical adhesive is applied on both lateral ends of the tape, not on the wound. This is how it is possible to apply it to longer and tense wounds. Furthermore, epithelialization is not affected by the liquefied bond because it is not applied directly to the wound edge. Despite these great advantages, few articles related to the clinical application of Leukosan SkinLink have been published [5].

In facial laceration, the main concern is the resulting scar. Scar evaluation using the Vancouver Scar Scale has been performed in many studies. However, the scale was originally developed for evaluating burn scars and is limited by its inability to evaluate subjective symptoms that patients experience such as itching and pain [6]. In this study, we used the POSAS for not only the physician's assessment, but also the patient's subjective symptoms at the same point in time. It is a useful and reliable tool that contains evaluation items for both patients and observers, and it can be applied to both burn and linear scars $[2,7]$. One month after surgery in this study, when scar remodeling had not begun, the physicians' assessments in the experimental group seemed to be worse because the eversion and the approximation of the wound edge was not as precise as in the control group. However, six months after surgery, when scar maturation had begun, there was no statistically significant difference between the groups.

It turned out that both the total operation time and the operation time per wound centimeter with sedation were shorter in the experimental group. Using Leukosan SkinLink shortened the operating time by 15 minutes on average for each wound centimeter, compared to conventional suturing. The average satisfaction score for the operation process was 2.9 points for the control group and 3.19 points for the experimental group. It is statistically higher in the experimental group. Juergens et al. [5] also reported less total operative time and less time for the period from anesthesia to suture in cesarean incision wounds closed using Leukosan SkinLink. In this study, when facial laceration patients were treated with Leukosan SkinLink, they were satisfied with the surgical process and the much shorter operation time. Additionally, there was no difference in the final clinical and aesthetic outcomes.

One patient presented with pruritic rash and papules in and around the Leukosan SkinLink. The authors did not perform the patch test to determine the allergen, but it is thought that 2-octyl cyanoacrylate in the liquefied adhesive was the allergen because dermatitis only broke out on the area in direct contact with the liquefied adhesive, not in the area covered by the tape. This should be avoidable with minimal application of the liquefied adhesive on the tape [8].

There are some limitations in this study. First, Leukosan SkinLink is not suitable for all wounds. Adhesive tape does not adhere well to the mucosa or regions with hair or joints, and it is difficult to use adhesive tape to properly adjust the edges of wounds torn into multiple sections or located around skin defects and covered with flaps. Second, the POSAS is more objective than other scar scales for scar evaluation owing to evaluation from both physicians and patients, but the fact remains that it is a subjective tool. To improve the objectivity of the study, measuring equipment such as cutometers, durometers, and chromameters could have been used, but time and cost needed to be taken into account [9]. Third, we were unable to eliminate the bias inherent in allowing patients and guardians to choose the closure materials.

In summary, when using Leukosan SkinLink to treat simple facial laceration in the emergency department, we successfully reduced the treatment time and the use of sedatives and local anesthetics compared to conventional suturing, with no significant difference in cosmetic appearance. Therefore, Leukosan SkinLink can be used as the main suturing material for primary wound closure for patients with facial laceration who are unconscious due to drunkenness or head trauma, those who are vitally unstable due to multiple severe traumas, and children in particular, who tend to be uncooperative. The treatment method produced satisfying results for both patients and surgeons.

\section{REFERENCES}

1. Kang SY, Lee W, Cho SH, et al. Application of 2-Octylcyanoacrylate Dermabond(R) for Laceration Repair in Emergency Department. J Korean Soc Plast Reconstr Surg 2004; 31:337-42. 
2. Draaijers LJ, Tempelman FR, Botman YA, et al. The patient and observer scar assessment scale: a reliable and feasible tool for scar evaluation. Plast Reconstr Surg 2004;113:1960-5.

3. Islam S, Ansell M, Mellor TK, et al. A prospective study into the demographics and treatment of paediatric facial lacerations. Pediatr Surg Int 2006;22:797-802.

4. Kim JH, Kwon SB, Eo SR, et al. Convenient suture technique for pediatric facial lacerations. J Korean Soc Plast Reconstr Surg 2010;37:496-8.

5. Juergens S, Maune C, Kezze F, et al. A randomized, controlled study comparing the cosmetic outcome of a new wound closure device with Prolene suture closing caesarean wounds.
Int Wound J 2011;8:329-35.

6. Fearmonti R, Bond J, Erdmann D, et al. A review of scar scales and scar measuring devices. Eplasty 2010;10:e43.

7. van de Kar AL, Corion LU, Smeulders MJ, et al. Reliable and feasible evaluation of linear scars by the patient and $\mathrm{Ob}$ server Scar Assessment Scale. Plast Reconstr Surg 2005;116: 514-22.

8. Bowen C, Bidinger J, Hivnor C, et al. Allergic contact dermatitis to 2-octyl cyanoacrylate. Cutis 2014;94:183-6.

9. Widgerow AD, Chait LA, Stals PJ, et al. Multimodality scar management program. Aesthetic Plast Surg 2009;33:533-43. 